\title{
Modeling of the Collector Epilayer of a Bipolar Transistor in the MEXTRAM Model
}

\author{
H. C. de Graaff and W. J. Kloosterman
}

\begin{abstract}
A new model description for the behaviour of epitaxial collectors in bipolar transistors is given. This is part of MEXTRAM, a compact model for circuit simulation, and it gives the voltage drop and stored minority carrier charge in the collector epilayer as a function of the bias conditions. It covers (total) depletion and quasi-saturation for both ohmic and space charge conditions in the end region of the epilayer. New features are that the collector current and stored charge are given as explicit analytical functions, thus guaranteeing continuity also for their derivatives, and that collector current spreading is taken into account.
\end{abstract}

\section{INTRODUCTION}

A MONG the bipolar compact models for circuit design the Gummel-Poon model [1] is up to now widely used. In its extended Spice versions quasi-saturation or base push-out effects are modeled by a modification of $Q_{b e}$, the stored charge of minority carriers injected from the emitter into the base.

Kull et al. [2] improved the Gummel-Poon model in a more realistic and physical way by modeling the voltage drop and the charge storage in the epitaxial collector region in the quasisaturation situation. However, their analysis was based on the assumption of quasi-neutrality in the entire epitaxial collector region, thus excluding voltage drops due to space charge modulation by hot carriers. It is true that the Kull model has a mobility depending on the quasi-Fermi level gradient, but the formulation gives erroneous results for $I_{c}>I_{h c}=q \cdot N \cdot v_{s} \cdot A_{e}$ (see e.g. Fig. 6).

Jeong and Fossum [3] extended the Kull model for $I_{c}>I_{h c}$ by incorporating hot-carrier modulation of the space charge. They approximated the drift velocity of the carriers in the epilayer as a function of the electric field by two straight lines: $v_{d}=\mu \cdot E$ and $v_{d}=v_{s}$, with $\mu$ and $v_{s}$ as constants. This means physically that only purely ohmic behavior and complete velocity saturation are considered. It also implies that in the crossover point the derivatives of the function are discontinuous, leading to numerical instabilities. Moreover, the solution required an iterative procedure.

In the first version of the MEXTRAM model [4] the hotcarrier effects in the collector epilayer were also taken into account. The formulation was such that a smooth transition from ohmic to hot-carrier situations was guaranteed and the

Manuscript received June 21, 1994; revised September 2, 1994. The review of this paper was arranged by Associate Editor T. Nakamura.

H.C. de Graaff is with the Department of Electronic Engineering, Delft University of Technology, $2600 \mathrm{GA}$, Delft, The Netherlands. He is also with the University of Twente, Enschede, The Netherlands.

W. J. Kloosterman is with Philips Research Laboratories, 5656 AA Eindhoven, The Netherlands.

IEEE Log Number 9407526. internal base-collector voltage $V_{j}$ was written as an explicit analytical function of $I_{c}$ and $V_{c b}$. The drawback was that this $V_{j}$ is not the internal base-collector junction voltage in quasi-saturation when $I_{c}>I_{h c}$. A consequence was that this $V_{j}$, although it gave the right answer for the epilayer charge storage, could not be used for the collector depletion charge and a second internal junction voltage was needed.

In this paper we propose a new formulation of the voltage drop and the charge storage in the epitaxial collector region, more suitable to be implemented in a compact transistor model. It gives the collector current $I_{c}$ and the stored charge $Q_{e p i}$ in explicit form, without discontinuities and for all bias situations (ohmic, hot carriers, total depletion and even reverse mode of operation). The internal voltage $V_{j}$ is valid for both depletion and charge storage. Three-dimensional effects (sidewalls and current spreading) are incorporated without complicating the numerical solutions. For D.C. characteristics the results are similar to those of the Jeong-Fossum model, but the numerical stability is much greater. Because the higher derivatives are continuous, non-linear distorsion is fairly well predicted [12]. At low current levels (ohmic behavior) it is identical with the Kull model, but much more realistic at higher current levels (see Fig. 6).

The model has been implemented in a new release of MEXTRAM [13] and tested in the circuit simulator Pstar (used within the Philips' designers community) and in the Hewlett-Packard's Microwave Design System.

\section{ONE-DIMENSIONAL DESCRIPTION}

Basically we can distinguish four different situations in the collector epilayer of a bipolar transistor in the forward mode of operation. These are characterized by the electric field distributions in the epilayer, which are sketched in Figs. 1-4 for an npn transistor.

\section{A. Depletion Plus Ohmic}

The base-collector junction is situated at $x=0$ and $W$ is the width of the epitaxial layer (see Fig. 1); the width of the depletion layer $x_{d}$ is given by [5]

$$
x_{d}=x_{d_{0}} \cdot \sqrt{\frac{1+V_{j} / V_{d}}{1-I_{c} / I_{h c}}}
$$

with $x_{d_{0}}=\sqrt{\left(2 \cdot \epsilon \cdot V_{d}\right) /(q \cdot N)}$. In deriving (1) it is assumed that the base-collector junction is abrupt and asymmetrical 




Fig. 1. Electric field distribution in the epitaxial collector for the "depletion plus ohmic" situation. $W$ is the epitaxial layer thickness, $x_{d}$ the depletion layer thickness.

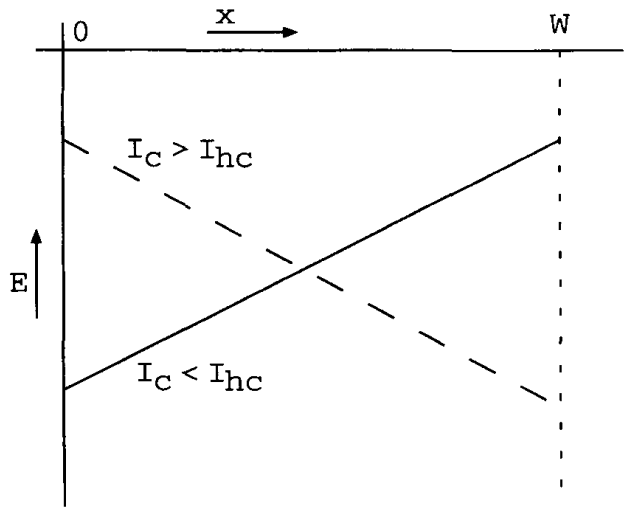

Fig. 2. Electric field distribution when total depletion occurs.

$\left(N_{\text {base }} \gg N\right) . V_{j}$ is the internal junction voltage, given by

$$
V_{j}=V_{c b}-I_{c} \cdot R_{c v}
$$

Equation (2) represents the ohmic voltage drop in the epilayer when a collector current $I_{c}$ flows. $R_{c v}$ is the ohmic resistance of the whole epilayer:

$$
R_{c v}=\frac{\rho \cdot W}{A_{e}}=\frac{W}{q \cdot \mu_{n} \cdot N \cdot A_{e}}
$$

Here $N$ is the epilayer dope, assumed to be constant, and $A_{e}$ is the emitter area.

In the depletion region $\left(0-x_{d}\right)$ we assume the electric field to be high enough to cause saturation of the drift velocity $\left(v_{d r}=v_{s}\right)$. The term $\left(1-I_{c} / I_{h c}\right)$ in (1) then represents the modulation of the space charge by the electrons in transit $\left(I_{c} /\left(q \cdot A_{e} \cdot v_{s}\right)\right)$. The quantity $I_{h c}$ is related to the fixed charge of the ionized donors: $I_{h c}=q \cdot N \cdot v_{s} \cdot A_{e}$.

\section{B. Total Depletion}

When in (1) $V_{j}$ increases and/or $I_{c}$ approches $I_{h c}, x_{d}$ may become equal to $W$ : the epilayer is then totally depleted. If

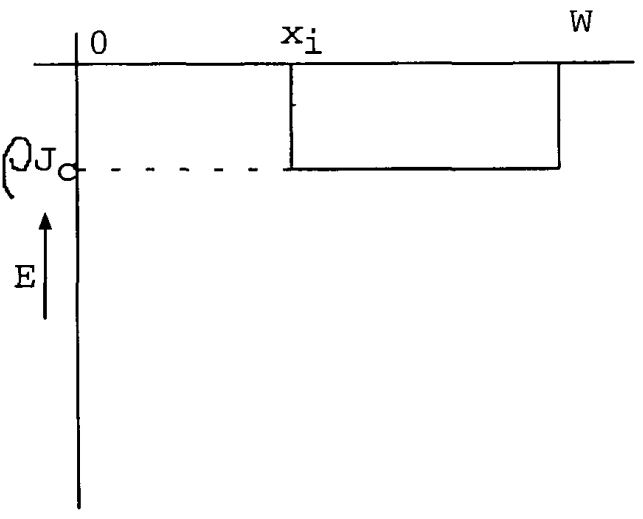

Fig. 3. Electric field distribution for the "injection plus ohmic" situation The injection region is from $x=0$ to $x=x_{i}$.



Fig. 4. Electric field distribution for the "injection plus hot carrier" situation $E\left(x_{i}\right)=-v_{s} / \mu_{n}$.

$I_{c}>I_{h c}$ the mobile electron charge overcompensates the fixed impurity charge $N$ and the field gradient changes sign. This is sketched in Fig. 2.

\section{Injection Plus Ohmic}

From (1) and (2) it follows that $x_{d}=0$ if $V_{c b}+V_{d}-I_{c} \cdot R_{c v}=$ 0 . This means that the base-collector junction is no longer reversely biased and a further increase in $I_{c}$ (or a decrease in $V_{c b}$ ) causes injection of holes from the base into the collector epilayer. These holes will be stored in the injection region $\left(0<x<x_{i}\right)$, see Fig. 3. If we assume that in this injection region there is no recombination $\left(J_{p}=0\right)$ and quasi-neutrality $(N+p=n)$ [6], [7] we can write for the majority carrier current density $J_{c}$ :

$$
J_{c}=q \cdot D_{n} \cdot\left(2+\frac{N}{p}\right) \cdot \frac{d p}{d x}
$$

Now we introduce normalized concentrations for $p(0), p\left(x_{i}\right)$ and $p(w)$ :

$$
p_{0, i, w}=\frac{p\left(0, x_{i}, w\right)}{N}
$$


Using the neutrality condition we can write for the respective pn-products:

$$
\begin{aligned}
p_{0} \cdot\left(p_{0}+1\right) & =\exp \left\{-\left(V_{j}+V_{d}\right) / V_{t}\right\} \\
p_{i} \cdot\left(p_{i}+1\right) & =\exp \left\{-\left(V_{i}+V_{d}\right) / V_{t}\right\} \\
p_{w} \cdot\left(p_{w}+1\right) & =\exp \left\{-\left(V_{c b}+V_{d}\right) / V_{t}\right\}
\end{aligned}
$$

$V_{t}$ is the thermal voltage $k \cdot T / q$ and $V_{d}$ is the diffusion voltage $\left(V_{d}=V_{t} \cdot \ln \left(N / n_{i}\right)^{2}\right)$. Following Kull et al. [2] we introduce the function $K(V)$

$$
K(V)=\sqrt{1+4 \cdot \exp \left\{-\left(V+\overline{V_{d}}\right) / V_{t}\right\}}
$$

and get

$$
\begin{aligned}
& K_{0, i, w}=K\left(V_{j}, V_{i}, V_{c b}\right)=2 \cdot p_{0, i, w}+1 \\
& V_{j, i, c b}=-V_{d}-V_{t} \cdot \ln \frac{K_{0, i, w}^{2}-1}{4} .
\end{aligned}
$$

The voltages $V_{j, i, c b}$ are the local differences between the quasi Fermi levels $\varphi_{n}$ and $\varphi_{p}$. With these normalized concentrations we can integrate (4) over the injection region:

$$
J_{\mathrm{c}}=\frac{q \cdot D_{n} \cdot N}{x_{i}} \cdot\left(2 \cdot p_{0}-2 \cdot p_{i}+\ln \frac{p_{0}}{p_{i}}\right)
$$

Substituting (7) and (8) and after multiplying both sides by the emitter area $A_{e}$ we get

$$
\begin{aligned}
I_{c} & =\frac{V_{0 i}+V_{i}-V_{j}}{x_{i} / W \cdot R_{c v}} \\
V_{0 i} & =V_{t} \cdot\left(K_{0}-K_{i}-\ln \frac{K_{0}+1}{K_{i}+1}\right) .
\end{aligned}
$$

In (10) the voltage $V_{j}$ is again considered as the internal junction voltage and $V_{i}-V_{j}$ is then the small voltage drop across the injection region.

As the end region $\left(x_{i}<x<W\right)$ is supposed to be ohmic, with $J_{p}=0$ and $p+N=n$, (4) can also be integrated from $x_{i}$ to $W$, leading finally to an alternative formulation of $(10)$ :

$$
\begin{aligned}
I_{c} & =\frac{V_{i w}+V_{c b}-V_{i}}{\left(1-x_{i} / W\right) \cdot R_{c v}} \\
V_{i w} & =V_{t} \cdot\left(K_{i}-K_{w}-\ln \frac{K_{i}+1}{K_{w}+1}\right)
\end{aligned}
$$

$V_{c b}-V_{i}$ is here the voltage drop across the ohmic end region.

The model of Kull et al. is obtained when (4) is integrated over the whole epilayer, from 0 to $W$ and by adding an extra term to account for non-ohmic behavior:

$$
\begin{aligned}
I_{c} & =\frac{V_{0 w}+V_{c b}-V_{j}}{R_{c v}+\left(V_{c b}-V_{j}\right) / I_{h c}}, \\
V_{0 w} & =V_{t} \cdot\left(K_{0}-K_{w}-\ln \frac{K_{0}+1}{K_{w}+1}\right)
\end{aligned}
$$

Note that $(10)$ is generally applicable whenever an injection region exists, in contrast with (11) and (12), which also require the existence of an ohmic end region.

\section{Injection Plus Hot Carriers}

For $I_{c}>I_{h c}$ the end region $\left(x_{i}<x<W\right)$ no longer shows ohmic behavior; the injection region is followed by a space charge region filled with hot electrons: see Fig. 4.

Poisson's equation reads as [7]:

$$
\frac{d E}{d x}=\frac{q \cdot N}{\epsilon}\left(1-\frac{I_{c}}{I_{h c}}\right)
$$

We integrate this twice over the region from $x_{i}$ to $W$ and get, with $E\left(x_{i}\right)=-v_{s} / \mu_{n}$ as boundary condition

$$
I_{c}=I_{h c}+\frac{V_{c b}-V_{i}-I_{h c} \cdot R_{c v} \cdot\left(1-x_{i} / W\right)}{H R_{c v} \cdot\left(1-x_{i} / W\right)^{2}}
$$

where $H R_{c v}=W^{2} /\left(2 \cdot \epsilon \cdot v_{s} \cdot A_{e}\right)$ is the hot carrier epilayer resistance. Usually is $H R_{c v}>R_{c v}$. The quantity $x_{i} / W$ follows from (10). As an extra condition to solve for $V_{i}$ we now require that the electric field is continuous at $x=x_{i}$, equals $-v_{s} / \mu_{n}$ of the injection region and space charge region. Finally, this leads to (see Appendix A)

$$
V_{i}=-V_{d}-V_{t} \cdot \ln \frac{\left(I_{c} / I_{h c}\right)^{2}-1}{4} .
$$

Equations (10), (13), and (14) establish a relationship between $I_{c}, V_{c b}$ and $V_{j}$, so the problem is in principle solved. So far the treatment is simular to that of Jeong and Fossum [3] but for some details. The relationship is not well suited for being part of a compact model for circuit simulation for it needs an iterative procedure and it possess discontinuous derivatives at the transition point $I_{c}=I_{h c}$. We will simplify the model by the following approximations;

1) From (7), (10), (11), and (12) we obtain

$$
V_{0 i}-V_{0 w}=V_{t} \cdot\left(2 \cdot p_{w}-2 \cdot p_{i}+\ln \frac{p_{i}+1}{p_{w}+1}\right) .
$$

In all practical cases we have either $p_{w} \ll 1$ and $p_{i} \ll 1$ (normal forward mode) or $p_{i} \approx p_{w}$ (hard saturation or inverse mode of operation). So, approximately $V_{0 i} \approx$ $V_{0 w}$ and $V_{i w}=V_{0 w}-V_{0 i} \approx 0$.

2) From (5) and (14) we have

$$
\begin{aligned}
& V_{j}=-V_{d}-V_{t} \cdot \ln \left\{p_{0} \cdot\left(p_{0}+1\right)\right\} \approx-V_{d} \\
& V_{i}=-V_{d}-V_{t} \cdot \ln \left\{\frac{\left(I_{c} / I_{h c}\right)^{2}-1}{4}\right\} \approx-V_{d} .
\end{aligned}
$$

So, roughly, $V_{i} \approx V_{j}$

With the approximations 1. and 2. (13) becomes

$$
I_{c} \approx I_{h c}+\frac{V_{c b}-V_{j}-I_{h c} \cdot R_{c v} \cdot\left(1-x_{i} / W\right)}{H R_{c v} \cdot\left(1-x_{i} / W\right)^{2}}
$$

and (10) changes into

$$
\frac{x_{i}}{W} \approx \frac{V_{0 w}}{I_{c} \cdot R_{c v}} .
$$

Equations (15) and (16) give $I_{c}$ implicitly as a function of $V_{j}$ and $V_{c b}$ in the injection plus hot carrier situation. Fig. 5 compares the exact solution with the approximated model. In this picture we use a regional model below and above $I_{h c}$ with discontinuous derivatives at the transition point. This will be eliminated in the next section. 




Fig. 5. Comparison between the exact solution (full model) of (10), (13), and (14) and the approximation of (15) and (16). A smooth transition between ohmic and hot carrier situations is not yet depicted.

Merging the ohmic and hot carrier formulas: In situation C. (injection plus ohmic) we will call $I_{c}$ now $I_{\Omega}$ and choose (11) together with the approximations 1 . and 2., as the appropriate expression:

$$
I_{\Omega}=\frac{V_{i w}+V_{c b}-V_{i}}{\left(1-x_{i} / W\right) \cdot \bar{R}_{c v}} \approx \frac{V_{c b}-V_{j}}{\left(1-x_{i} / W\right) \cdot R_{c v}}
$$

We define a current $I_{\text {low }}$ as

$$
I_{\text {low }}=\frac{I_{\Omega} \cdot I_{h c}}{I_{\Omega}+I_{h c}}
$$

which sets a limit $\left(I_{h c}\right)$ to $I_{\Omega}$. If we transform the current $I_{c}$ in (15) into

$$
I_{c}=I_{l o w}+\frac{I_{\Omega}-I_{l o w}}{1-x_{i} / W} \cdot \frac{R_{c v}}{H R_{c v}}
$$

we get a smooth transition from $I_{c}=I_{\Omega}$ for $I_{\Omega} \ll I_{h c}$ to

$$
I_{c}=I_{h c}+\frac{V_{c b}-V_{j}-I_{h c} \cdot R_{c v} \cdot\left(1-x_{i} / W\right)}{H R_{c v} \cdot\left(1-x_{i} / W\right)^{2}}
$$

for $I_{\Omega} \gg I_{h c}$. Together with $x_{i} / W=V_{0 w} /\left(I_{c} \cdot R_{c v}\right)$ from (16), (17), (18), and (19) give $I_{c}\left(V_{j}, V_{c b}\right)$ for all four situations $\mathrm{A}, \mathrm{B}, \mathrm{C}$ and $\mathrm{D}$. If injection does not occur (situation $\mathrm{A}$ and B) $V_{0 w}=0$ and $x_{i}=0$, but (19) remains valid, as the reader may easily verify.

In Fig. 6 the behavior of the MEXTRAM, Kull and JeongFossum models are shown. The MEXTRAM results differ around $I_{c} \approx I_{h c}$ appreciable from those of the Jeong-Fossum model and we derive an explicit function, without solving a set of non-linear equations. Moreover, their solution shows discontinuities in the first and higher order derivatives at



Fig. 6. Normalized collector current $\left(I_{c} / I_{h c}\right)$ versus the normalized epilayer voltage drop $\left(V_{c b}-V_{j}\right) /\left(I_{h c} \cdot R_{c e}\right)$ for the MEXTRAM, Kull and Jeong-Fossum models.

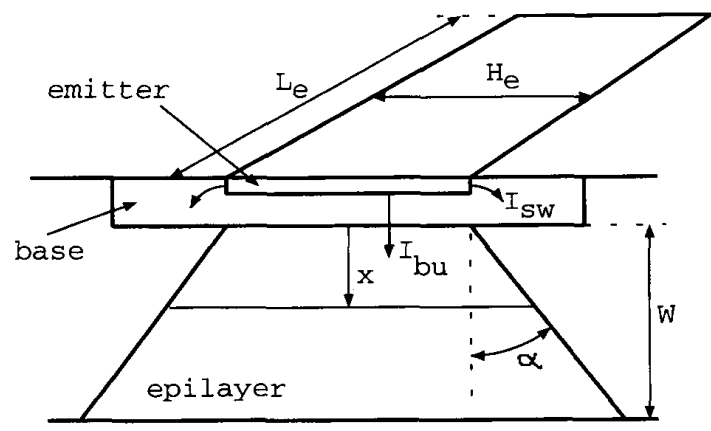

buried layer

Fig. 7. Three-dimensional sketch of the transistor structure. Emitter dimensions are $L_{\epsilon} \times H_{\epsilon}, \alpha$ is the current spreading angle.

$I_{c}=I_{h c}$, caused by the drift velocity not being an analytical function of the electric field.

\section{ThreE-DimENSIONAL EXTENSION}

In this section we will account for sidewall effects and for the current spreading in the collector epilayer in a simple and approximative way. The situation is sketched in Fig. 7: $L_{e}$ and $H_{e}$ are the emitter dimensions, $\alpha$ is the spreading angle.

The total collector current $I_{c}$ consist of the bulk and the sidewall components $\left(I_{b u}=J_{b u} \cdot H_{e} \cdot L_{e}, I_{s w}=2 \cdot J_{s w}\right.$. $\left(H_{e}+L_{e}\right)$ ) so:

$$
I_{b u}=\frac{I_{c}}{1+2 \cdot Y_{c} \cdot\left(\frac{1}{H_{e}}+\frac{1}{L_{e}}\right)}
$$


where $Y_{c}=J_{s w} / J_{b u}$ is the ratio of the sidewall and bulk current densities [14]. Due to the current spreading the bulk current density $J_{b u}$ becomes position-dependent:

$$
\begin{aligned}
J_{b u}(x) & =\frac{I_{c}}{A_{e} \cdot\left\{1+2 \cdot Y_{c}\left(\frac{1}{H_{e}}+\frac{1}{L_{e}}\right)\right\} \cdot(1+a \cdot x)} \\
a & =2 \cdot\left(\frac{1}{H_{e}}+\frac{1}{L_{e}}\right) \tan \alpha
\end{aligned}
$$

The ohmic resistance of the total epilayer now becomes

$$
R_{c v}=\frac{1}{I_{c}} \cdot \int_{0}^{W} \rho \cdot J_{b u}(x) d x .
$$

We may put

$$
R_{c v}=\frac{\rho \cdot W}{A_{e f f}}
$$

with

$$
\begin{aligned}
A_{e f f}= & \frac{a \cdot W \cdot A_{e} \cdot\left\{1+Y_{c} \cdot\left(\frac{1}{H_{e}}+\frac{1}{L_{e}}\right)\right\}}{\ln (1+a \cdot W)} \\
\approx & \left(1+\frac{1}{2} \cdot a \cdot W\right) \\
& \cdot A_{e} \cdot\left\{1+Y_{c} \cdot\left(\frac{1}{H_{e}}+\frac{1}{L_{e}}\right)\right\} .
\end{aligned}
$$

With respect to the one-dimensional case, the effective emitter area has increased and $R_{c v}$ has decreased. From the comparison with exact spreading resistance calculations [8] we learn that the angle $\alpha \approx 40^{\circ}$.

We return to situation D (injection plus hot carriers, see Fig. 4). Poisson's equation in the end region $\left(x_{i}<x<W\right)$ now reads as

$$
\frac{d E}{d x}=\frac{q \cdot N}{\epsilon} \cdot\left\{1-\frac{I_{\varepsilon}}{I_{h c} \cdot(1+a \cdot x)}\right\} .
$$

Integrating twice again gives

$$
I_{c}=S \cdot\left\{I_{h c}+\frac{V_{c b}-V_{i}-\frac{v_{s}}{\mu_{n}} \cdot W \cdot\left(1-x_{i} / W\right)}{H R_{c v} \cdot\left(1-x_{i} / W\right)^{2}}\right\}
$$

The difference with (13) in the one-dimensional case is the multiplication with the spreading factor $S$. For this function $S$ we get, after rather extensive mathematical manupulations (see appendix B),

$$
S \approx 1+\frac{1}{3} \cdot a \cdot W \cdot\left(1+2 \cdot \frac{x_{i}}{W}\right)
$$

Although this approximation is derived for $a \cdot W<1$, it is also reasonably good for $a \cdot W>1$, see Fig. 8 .

This $S$ differs from the spreading factor $(1+(1 / 2) \cdot a \cdot W)$ in the ohmic case (23) in that

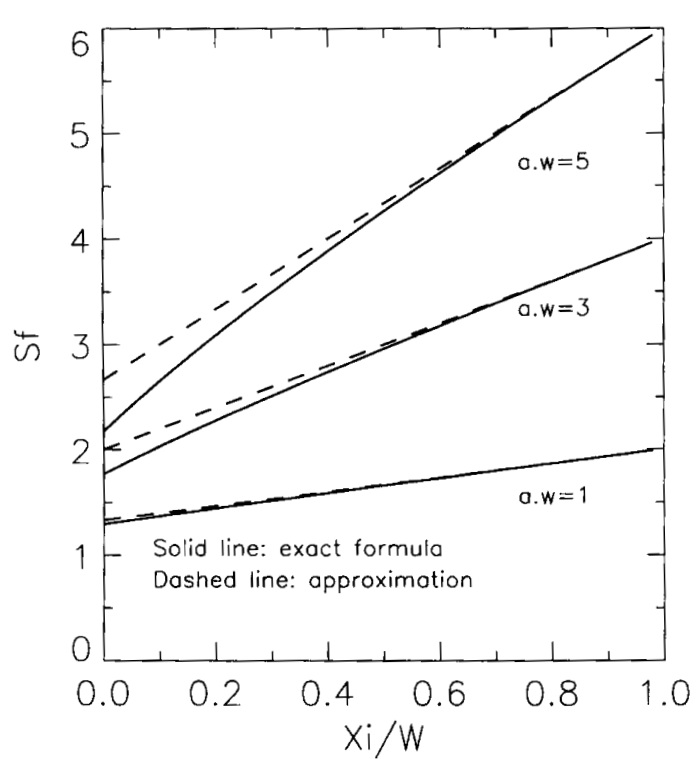

Fig. 8. Comparison between the exact spreading factor and the approximation (26) for large values of $a \cdot W$.

- it depends on $x_{i}$ : for $x_{i}=0, S \approx 1+1 / 3 \cdot a \cdot W$

- the spreading angle is usually larger than $40^{\circ}$ at these high current levels above $I_{h c}$

We follow the same procedure as in the one-dimensional case and change in (25) $V_{i}$ into $V_{j}$, define $I_{\Omega}$ and $I_{l o w}$ as in (17) and (18) and take $S \cdot I_{h c} \approx I_{l o w}$ and $v_{s} \cdot W / \mu_{n} \approx I_{h c} \cdot R_{c v}$. Then we end up with

$$
I_{c}=I_{l o w}+\left(1+b \cdot x_{i} / W\right) \cdot \frac{I_{\Omega}-I_{l o w}}{1-X_{i} / W} \cdot \frac{R_{c v}}{S C R_{c v}}
$$

with

$$
\begin{aligned}
x_{i} / W & =\frac{V_{0 W}}{I_{c} \cdot R_{c v}} \\
b & =\frac{2 \cdot a \cdot W}{3+a \cdot W} \\
S C R_{c v} & =\frac{H R_{c v}}{1+1 / 3 \cdot a \cdot W} .
\end{aligned}
$$

The main differences with (19) are the extra factor $1+b$. $x_{i} / W, S C R_{c v}$ replacing the $H R_{c v}$ and $R_{c v}$ being defined by (22) instead of (3).From this three dimensional derivation we see how the epilayer parameters scale with the device geometry. The dominant effect is $R_{c v}$ and $S C R_{c v}$ having their 3-D values; the factor $\left(1+b \cdot x_{i} / W\right)$ is of minor importance. However, when avalanche multiplication has to be implemented in a complete transistor model, the spreading parameter $b$ is very important, because the maximum electric field resides at the buried layer and is strongly decreased by this current spreading. But this is beyond the scope of this paper and will not be treated.

The 5 parameters of the formula $I_{c}\left(V_{j}, V_{c b}\right)$ are $R_{c v}-$ $S C R_{c v}-I_{h c}-V_{d}$ and $b$. 


\section{Charge Storage IN THE EPILAYER}

In the situations "injection plus ohmic" (C) and "Injection plus hot carriers" (D) we have a stored charge of minority carriers in the injection region:

$$
Q_{e p i}=q \cdot A_{e} \cdot \int_{0}^{x_{2}} p \cdot d x
$$

To calculate $Q_{e p i}$ it is most convenient to use the Moll-Ross formula [9] twice: once with $Q_{e p i}$ included and once without $Q_{e p i}$

$$
I_{c}=Q_{b 0} \cdot I_{s} \cdot \frac{\exp \left(V_{b e} / V_{t}\right)-\exp \left(-V_{c b} / V_{t}\right)}{Q_{b 0}+Q_{T}+Q_{b}+Q_{e p i}}
$$

and

$$
I_{c}=Q_{b 0} \cdot I_{s} \cdot \frac{\exp \left(V_{b e} / V_{t}\right)-\exp \left(-V_{j} / V_{t}\right)}{Q_{b 0}+Q_{T}+Q_{b}} .
$$

In these formulas is $Q_{b 0}$ the fixed base charge, $Q_{T}$ the sum of the emitter and collector depletion charges and $Q_{b}$ the stored charge in the base. From the foregoing we can easily derive that

$$
Q_{e p i}=I_{s} \cdot Q_{b 0} \cdot \frac{\exp \left(-V_{j} / V_{t}\right)-\exp \left(-V_{c b} / V_{t}\right)}{I_{c}} .
$$

Near the origin of the $\left(I_{c}, V_{c e}\right)$ characteristics we have $V_{j} \approx$ $V_{c b}$ and $I_{c} \approx 0$, so (28) will cause numerical problems there. Substitution of (12) in (28) and taking $V_{j} \approx V_{c b}$ the stored epilayer charge becomes

$$
Q_{e p i}=R_{c v} \cdot I_{s} \cdot Q_{b 0} \cdot \exp \left(V_{d} / V_{t}\right) \cdot \frac{p_{0}+p_{2}}{2 \cdot V_{t}}
$$

where use has been made of (5), (7) and (8). Equations (28) and (29) require no additional parameters; $Q_{b 0}$ is already a given MEXTRAM parameter [4].

\section{IMPLEMENTATION IN MEXTRAM}

The topology of the relevant parts in the equivalent circuit of MEXTRAM is sketched in Fig. 9. In this figure is $R_{c c}$ the constant resistance of the buried layer, $R_{b c}$ the constant resistance of the extrinsic base, $R_{b v}$ the bias-dependent resistance of the base under the emitter and $R_{e}$ the constant emitter series resistance. There are 5 internal nodes: $e_{1}-b_{1}-b_{2}-c_{1}$ and $c_{2} ; V c_{2} b_{2}$ replaces here the internal junction voltage $V_{j}$ of the preceding sections, $V c_{1} b_{2}$ corresponds with $V_{c b}$ and $I c_{1} c_{2}$ with $I_{c}$ in (27).

To solve for the epilayer current $I c_{1} c_{2}$ (27) we normalize with respect to $I_{h c}$ and introduce the following quantities:

$$
\begin{aligned}
s_{f} & =\frac{2 \cdot b}{1+b} \\
v & =\frac{V_{c b}-V_{j}}{I_{h c} \cdot R_{c v}}=\frac{V c_{1} b_{2}-V c_{2} b_{2}}{I_{h c} \cdot R_{c v}} \\
e & =\frac{V_{0 w}}{I_{h c} \cdot R_{c v}}=e\left(V c_{1} b_{2}, V c_{2} b_{2}\right)
\end{aligned}
$$

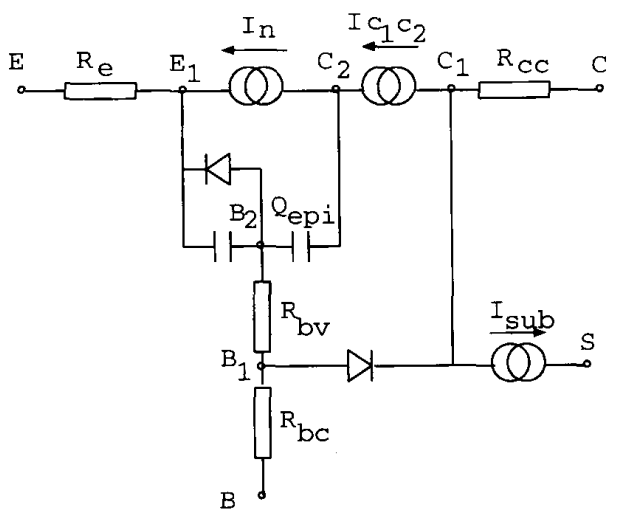

Fig. 9. Simplified topology of the MEXTRAM equivalent circuit.

$$
r=\frac{R_{c v}}{S C R_{c v}}
$$

Equation (27) is rewritten and finally we arrive at a cubic equation for $I c_{1} c_{2} / I_{h c}$ :

$$
\left(\frac{I c_{1} c_{2}}{I_{h c}}\right)^{3}+a_{2} \cdot\left(\frac{I c_{1} c_{2}}{I_{h c}}\right)^{2}+a_{1} \cdot\left(\frac{I c_{1} c_{2}}{I_{h c}}\right)+a_{0}=0
$$

with

$$
\begin{aligned}
& a_{2}=-2 \cdot e-\frac{v+r \cdot v^{2}+e}{1+v} \\
& a_{1}=\frac{e^{2} \cdot(3+v)+2 \cdot e \cdot v-s_{f} \cdot e \cdot r \cdot v^{2}}{1+v} \\
& a_{0}=\frac{e^{2} \cdot(e+v)}{1+v} .
\end{aligned}
$$

The solution for $I c_{1} C_{2}$ as a function of $V c_{1} b_{2}$ and $V c_{2} b_{2}$ is the real root of this cubic equation. If the current spreading parameter $b$ is put to zero, $s_{f}$ becomes zero, but in (30) only the coefficient $a_{1}$ will change somewhat. The epilayer stored charge $Q_{e p i}$ resides on a capacitor between the internal nodes $c_{2}$ and $b_{2}$, see Fig. 9. In the Kull's model [2] $Q_{e p i}$ is devided into a charge $Q_{0}$ between $c_{2}$ and $b_{2}$, depending on $p_{0}$, and a charge $Q_{w}$ between $c_{1}$ and $b_{2}$ depending on $p_{w}$. The difference with our topology (Fig. 9) is negligible because in the normal forward situation $Q_{w} \approx 0$ and in hard saturation and in the reverse mode of operation the voltage difference $V c_{1} c_{2} \approx 0$.

\section{PARAMETER ExTRACTION}

At the onset of quasi-saturation is $x_{i} / W$ still very small and the internal junction voltage $V_{j}$ is around the diffusion voltage. In this situation we can therefore substitute in (27) $x_{i} / W \approx 0$ and $V_{j} \approx-V_{d}$, leading to

$$
I_{c} \cdot R_{c v} \approx \frac{V_{c b}+V_{d}}{1+\frac{V_{c b}+V_{d}}{I_{h c} \cdot R_{c v}}} \cdot\left(1+\frac{R_{c v}}{S C R_{c v}} \cdot \frac{V_{c b}+V_{d}}{I_{h c} \cdot R_{c v}}\right) .
$$


If $\left(V_{c b}+V_{d}\right) \ll I_{h c} \cdot R_{c v}$, the ohmic case (C), we get

$$
I_{c} \approx \frac{V_{c b}+V_{d}}{R_{c v}}
$$

whereas in the hot carrier case (D) when $V_{c b}+V_{d} \gg I_{h c} \cdot R_{c v}$, we get

$$
I_{c} \approx I_{h c}+\frac{V_{c b}+V_{d}}{S C R_{c v}} .
$$

This onset of quasi-saturation can be determined from measuring the different bias points $\left(I_{c}, V_{c b}\right)$ where maxima of the low-frequency third harmonic distorsion occurs [10]. The parameters $R_{c v}$ and $V_{d}$ are then extracted for the ohmic case, and $I_{h c}$ and $S C R_{c v}$ for the hot carrier case. Apart from the third harmonic measurements, also the $h_{F E}$ and $f_{T}$ fall-off at high current can be used for extracting $R_{c v}, V_{d}, I_{h c}$ and $S C R_{c v}$. If the emitter dimensions $W_{e}$ and $L_{e}$, the epilayer thickness $W$ and epilayer dope $N$ are known, the parameters can be calculated and in most case fine tuning of $V_{d}$ and $R_{c v}$ is sufficient to describe accurately the transistor characteristics. The parameter $b$ can be obtained from the avalanche current at high collector currents: it determines the shape of the $I_{c}\left(V_{c e}\right)$ characteristics near the sustaining voltage $B V_{c e 0}$

\section{RESULTS}

The MEXTRAM model with the new collector epilayer description has been in use now for more than a year in our in-house circuit simulator Pstar. To illustrate its accuracy we will give here one example, where model calculations are compared with measurements. The transistor is a $12 \mathrm{GHZ}$ transistor, made in the Qubic process [11]. The epilayer model parameters for this transistor are: $I_{h c}=0.4 \mathrm{~mA}, R_{c v}=900$ $\Omega, S C R_{c v}=7000 \Omega, V_{d c}=720 \mathrm{mV}$ and the spreading factor $b=0.33$.

In Fig. 10 the $I_{c}\left(V_{c e}, I_{b}\right)$ characteristics are given, modeled as well as measured. Also indicated is the onset of quasisaturation (dashed line). One can see that a substantial part of the characteristics is in the quasi-saturation region. To illustrate the excellent scaling properties of this compact model, Fig. 11 shows the influence of the epilayer thickness on the collector current at a fixed bias point $\left(V_{c b}=0 \mathrm{~V}, V_{b e}=0.9 \mathrm{~V}\right)$ in the quasi-saturion. The compact model results are compared with Medici 1-D and 2-D device simulations of the same Qubic transistor from Fig. 10. We see that for $W_{e p i} \ll H_{e}$ there is hardly any difference between 1-D and 2-D results, but if $W_{e p i} \approx H_{\mathrm{e}}$ the 2-D current becomes about twice as high as the 1-D current. To decribe the 2-D Medici results the MEXTRAM epilayer compact model parameters are computed with $\tan (\alpha)$ $=1$ and $Y_{c}=0.1 \mu \mathrm{m}$. Both the 1-D and 2-D results can be described accurately with the MEXTRAM model. In Fig. 12 a simular comparison is made: now the emitter width $H_{e}$ is varied and the epilayer thickness $W_{e p i}$ and the emitter area $H_{e} \cdot L_{e}$ are kept constant. The same values for $\tan (\alpha)$ and $Y_{c}$ are used to compute the epilayer parameters. Here too there is excellent agreement between MEXTRAM and the Medici calculations. For large $H_{e}$ values the 2-D current

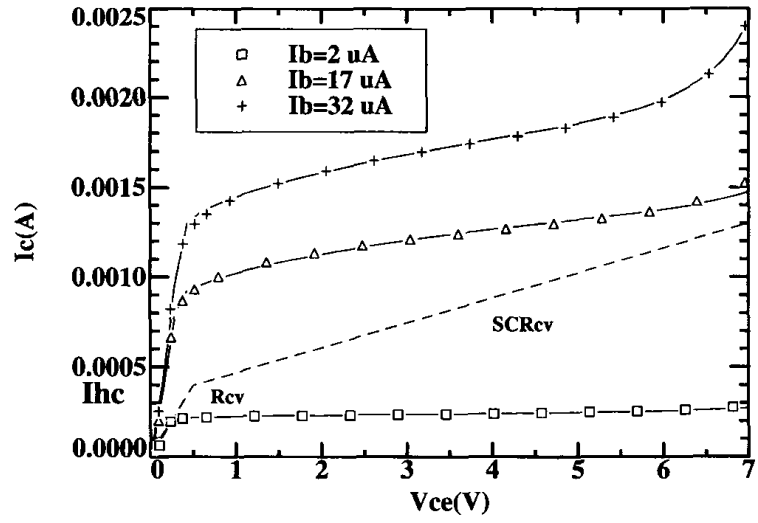

Fig. 10. Comparison between measured and modeled characteristics $I_{c}\left(V_{c e}\right)$ for 3 different values of $I_{b}$. dashed line: quasi-saturation onset.

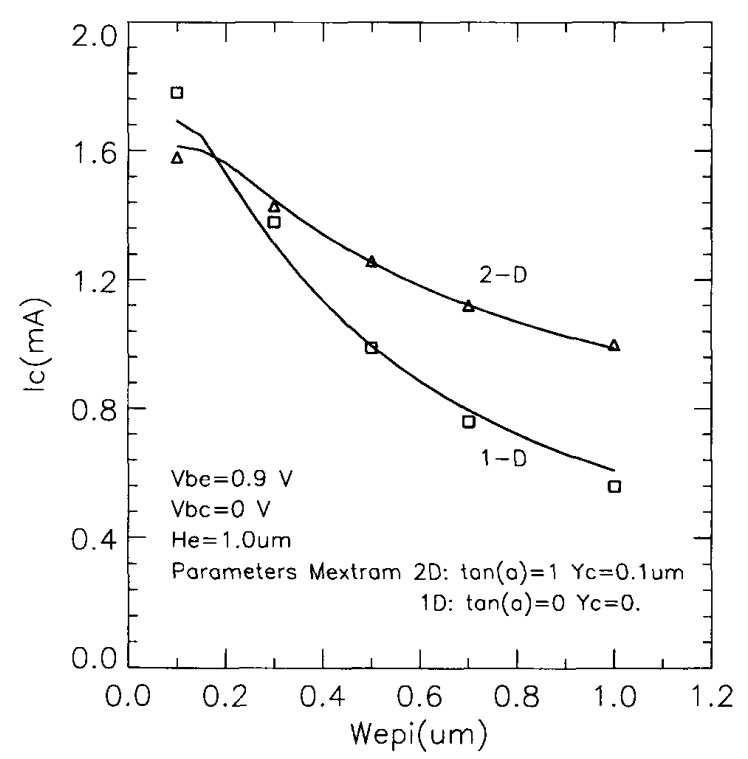

Fig. 11. Influence of the thickness of the epilayer on the collector current in quasi-saturation. Solid line: MEXTRAM model calculations. Markers: Medici 1D/2D device simulation.

comes below the 1-D current because of current crowding due to the increased base resistance.

\section{CONCLUSION}

A new compact model description for the behavior of epitaxial collectors in bipolar transistors is given. It substantially improves previous published models of Kull [2] and Jeong and Fossum [3]. The model of Kull is restricted to ohmic current flow and the model of Jeong and Fossum is implicit (formulated as a set of three nonlinear equations with three unknowns) and the first derivative is discontinuous, may lead to numerical instabilities in a circuit simulator. In our model the current through the epilayer is an explicit and continuous function of the internal and external base-collector junction voltages. The model covers all possible modes of operation 


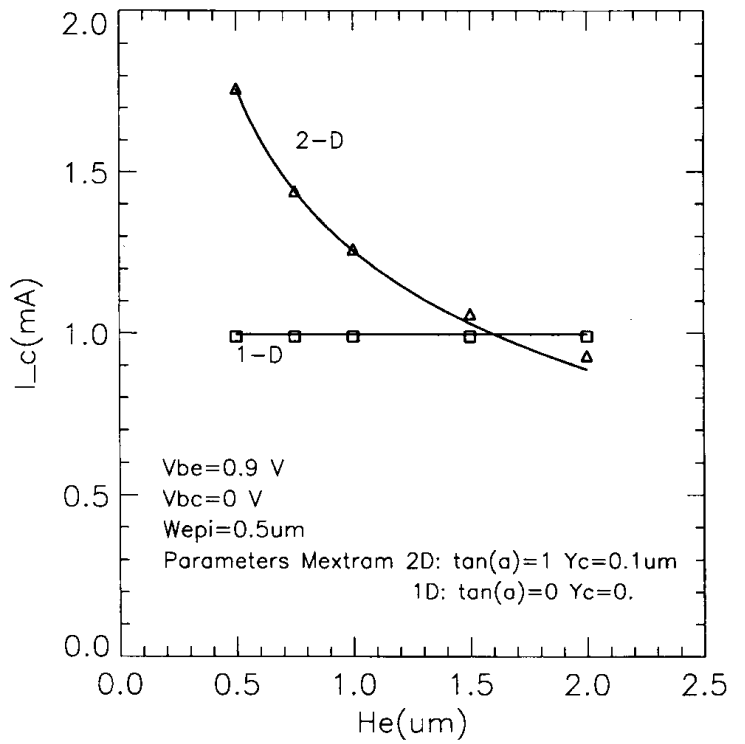

Fig. 12. Influence of the emitter width on the collector current in quasi-saturation. Solid line: MEXTRAM model calculations. Markers: Medici 1D/2D device simulation. The emitter area $H_{\epsilon} \cdot L_{\epsilon}$ is kept constant.

such as ohmic current flow, saturated current flow and base push out both in the forward and reverse mode of operation. The model is derived assuming one dimensional current flow. By comparison with 2-D device simulations it is shown that the change of the collector current due to variation of the emitter width and epilayer thickness can be described accurately by an appropriate scaling of the epilayer model parameters. These scaling rules are derived in the paper. The model has been implemented in a new release of MEXTRAM and is available for the public domain. Detailed information about the MEXTRAM model can be found in [13].

\section{APPENDIX A}

The electric field at $x=x_{i}$ is [6]:

$$
E\left(x_{i}\right)=\frac{k \cdot T}{q} \cdot \frac{1}{p} \cdot \frac{d p}{d x}
$$

Using (4) gives

$$
\begin{aligned}
E\left(x_{i}\right) & =-\frac{k \cdot T}{q} \cdot \frac{1}{p} \cdot \frac{I_{c}}{q \cdot D_{n} \cdot(2+N / p)} \\
& =-\frac{I_{c} \cdot R_{c v}}{W \cdot\left(1+2 \cdot p_{i}\right)} .
\end{aligned}
$$

So field continuity at $x=x_{i}$ then leads to

$$
\frac{v_{s}}{\mu_{n}}=\frac{I_{h c} \cdot R_{c v}}{W}=\frac{I_{c} \cdot R_{c v}}{W \cdot\left(1+2 \cdot p_{i}\right)}
$$

or

$$
\frac{I_{c}}{I_{h c}}=1+2 \cdot p_{i}=K_{i}=\sqrt{1+4 \cdot \exp \left\{-\left(V_{i}+V_{d}\right) / V_{t}\right\}}
$$

see (5) and (6).

\section{APPENDIX B}

The derivation of (26) goes as follows: we integrate (24) once and get, with $E\left(x_{i}\right)=-v_{s} / \mu_{n}$ as boundary condition:

$$
\begin{aligned}
E(x)= & -\frac{v_{s}}{\mu_{n}}+\frac{q \cdot N}{\epsilon} \cdot\left(x-x_{i}\right) \\
& -\frac{q \cdot N}{a \cdot \epsilon} \cdot \frac{I_{c}}{I_{h c}} \cdot \ln \frac{1+a \cdot x}{1+a \cdot x_{i}} .
\end{aligned}
$$

The voltage drop $\left(V_{c b}-V_{i}\right)$ is obtained from

$$
\begin{aligned}
V_{c b}-V_{i}= & -\int_{x_{i}}^{W} E(x) \cdot d x \\
= & \frac{v_{s}}{\mu_{n}} \cdot\left(W-x_{i}\right)-\frac{q \cdot N}{2 \cdot \epsilon} \cdot\left(W-x_{i}\right)^{2} \\
& +\frac{q \cdot N}{\epsilon} \cdot \frac{I_{c}}{I_{h c}} \cdot \frac{1+a \cdot x_{i}}{a^{2}} \\
& \cdot \int_{1}^{1+a \cdot W / 1+a \cdot x_{i}} \ln y \cdot d y \\
= & \frac{v_{s}}{\mu_{n}} \cdot\left(W-x_{i}\right)-\frac{q \cdot N}{2 \cdot \epsilon} \cdot\left(W-x_{i}\right)^{2} \\
& \cdot\left(1-\frac{I_{c}}{I_{h c}} \cdot S^{-1}\right) \cdot
\end{aligned}
$$

So the spreading function $S^{-1}$ is given by

$$
\begin{aligned}
S^{-1}= & \frac{2}{\left(W-x_{i}\right)^{2}} \cdot \frac{1+a \cdot x_{i}}{a^{2}} \cdot \int_{1}^{1+a \cdot W / 1+a \cdot x_{i}} \ln y \cdot d y \\
= & \frac{2 \cdot\left(1+a \cdot x_{i}\right)}{a^{2} \cdot\left(W-x_{i}\right)^{2}} \cdot\left\{\frac{1+a \cdot W}{1+a \cdot x_{i}} \cdot \ln \left(\frac{1+a \cdot W}{1+a \cdot x_{i}}\right)\right. \\
& \left.+1-\frac{1+a \cdot W}{1+a \cdot x_{i}}\right\} .
\end{aligned}
$$

We rewrite $(1+a \cdot W) /\left(1+a \cdot x_{i}\right)$ as $1+a \cdot\left(W-x_{i}\right) /\left(1+a \cdot x_{i}\right)$ and make a series expansion of the logarithm. For $a \cdot W$ (and $\left.a \cdot x_{i}\right)<1$ we then obtain

$$
\begin{aligned}
& S=\frac{1+a \cdot x_{i}}{1-\frac{a \cdot\left(W-x_{i}\right)}{3 \cdot\left(1+a \cdot x_{i}\right)}} \approx \frac{1+2 \cdot a \cdot x_{i}}{1-\frac{1}{3} \cdot a \cdot\left(W-4 \cdot x_{i}\right)} \\
& S \approx 1+\frac{1}{3} \cdot a \cdot W \cdot\left(1+2 \cdot \frac{x_{i}}{W}\right) .
\end{aligned}
$$

This is the approximation for $S$ as given in (26).

\section{ACKNOWLEDGMENT}

The authors wish to thank G. A. M. Hurkx for providing the Medici device simulations.

\section{REFERENCES}

[1] H. K Gummel and H. C. Poon, "An integral charge control model for bipolar transistors," Bell Syst. Techn. J., vol. 49, p.827, 1970.

[2] G. M. Kull, L. W. Nagel, S. W. Lee, P. LLoyd, E. J. Prendergast and H. Dirks, "A unified circuit model for bipolar transistors including quasisaturation effects," IEEE Trans. Electr. Dev.," vol. ED-32, p. 1103, 1985.

[3] H. Jeong and J. G. Fossum, "Physical modeling of high-current transistors for bipolar transistor circuit simulation," IEEE Trans. Electr. Dev., vol. ED-34, p. 898, 1987.

[4] H. C. de Graaff and F. M. Klaassen, Compact transistor modelling for circuit design. Wein, New York: Springer-Verlag, 1990 
[5] H. C. de Graaff, "Collector models for bipolar transistors," Solid-St. Electr., vol. 16, p. $587,1973$.

[6] J. R. A. Beale and J. A. G. Slatter, "The equivalent circuit of a transistor with a lightly doped collector operating in saturation," Solid-St. Electr. vol. 11, p. $241,1968$.

[7] H. C. de Graaff, "High current density effects in the collector of bipolar transistors," in Process and Device Modeling for Integrated Circuit Design, F v.d. Wiele, W. L. Engl, and P. G. Jespers eds., Noordhoff, Leiden, 1977.

[8] H. C. de Graaff, "Approximate calculations on the spreading resistance in multi-emitter structures," Philips Res. Repts., vol. 24, p. 34, 1969.

[9] J. L. Moll and J. M. Ross, "The dependence of transistor parameters on the distribution of base layer resistivity," Proc. IRE, vol. 44, p. 72, 1956.

[10] H. C. de Graaff and R. J. van der Wal, "Measurement of the onse of quasi-saturation in bipolar transistors," Solid-St. Electr, vol. 17, p. $1187,1974$.

[11] J. de Jong, R. Lane, B. van Schravendijk, and G. Conner, "Single polysilicon-layer advanced super high speed BICMOS technology," Proc. Bip. Circ. Techn. Meeting, paper 7.4, 1989.

[12] Submitted for the Bip. Circ. Techn. Meeting, 1994

[13] Nat.lab. Unclassified Report 006/94. The Mextram Bipolar Transistor Model. H. C. de Graaff, W. J. Kloosterman. Philips Research Laboratories. Prof. Holstlaan 4, 5656 AA Eindhoven, The Netherlands. (Request for a copy: Maarten Jan Officier, Electronic Design and Tools, Philips Reseach Laboratories, Prof. Holstlaan 4, 5656 AA Eindhoven.

[14] G. A. M. Hurkx, P. G. M. Baltus, E. P. M. Bladt, and M. P. G. Knuvers, "An efficient simulation method for linking bipolar process and device optimization to circuit performance," 1993 International Electron Device Meeting Technical Digest, paper 5.4, page 105.

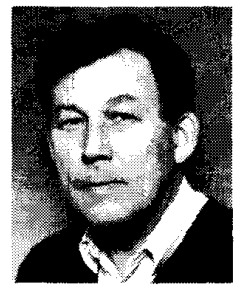

H. C. de Graaff was born in Rotterdam, The Netherlands, in 1933. He received the M.S. degree in electrical enineering from the Delft University of Technology, Delft, The Netherlands in 1956, and the Ph.D. degree from the University of Technology, Eindhoven, The Netherlands, in 1975

He joined Philips Research Laboratories, Eindhoven, in 1964, and has been working on thin film transistor, Most, bipolar devices, and materials research on polycrystalline silicon. His present field of interest is device modeling for circuit simulation. Since his retirement from Philips Research (Nov. 1991) he has been consultant to the University of Twente and the Technical University of Delft, both in The Netherlands.



W. J. Kloosterman was born in Olst, the Netherlands, in July 1951. He graduated from the Technical College of Zwolle in 1974.

He joined Philips Research Laboratories Eindhoven, The Netherlands, in 1974. He worked on the dynamical behavior of CRT tubes, powder com paction models, and, since 1980, has been involved in bipolar and MOS compact transistor modeling. characterization, and parameter extraction. 